\title{
Comparison of dry and gel based electrodes for P300 brain-computer interfaces
}

\author{
Christoph Guger ${ }^{1}$ *, Gunther Krausz ${ }^{1}$, Brendan Z. Allison ${ }^{\mathbf{2}}$ and Guenter Edlinger ${ }^{1}$ \\ g.tec medical engineering GmbH, Guger Technologies OG, Graz, Styria, Austria \\ ${ }^{2}$ Department of Cognitive Science, University of California at San Diego, La Jolla, California, USA
}

\section{Edited by:}

Cuntai Guan, Institute for Infocomm

Research, Singapore

\section{Reviewed by:}

Dennis J. McFarland, Wadsworth

Center for Laboratories and Research, USA

Michal Lavidor, Bar-llan University,

Israel

\section{*Correspondence:}

Christoph Guger, g.tec medical engineering $\mathrm{GmbH}$, Guger

Technologies OG, Herbersteinstrasse 60, A8010 Graz, Styria, Austria.

e-mail:guger@gtec.at
Most brain-computer interfaces (BCls) rely on one of three types of signals in the electroencephalogram (EEG): P300s, steady-state visually evoked potentials, and event-related desynchronization. EEG is typically recorded non-invasively with electrodes mounted on the human scalp using conductive electrode gel for optimal impedance and data quality. The use of electrode gel entails serious problems that are especially pronounced in real-world settings when experts are not available. Some recent work has introduced dry electrode systems that do not require gel, but often introduce new problems such as comfort and signal quality. The principal goal of this study was to assess a new dry electrode $\mathrm{BCl}$ system in a very common task: spelling with a $\mathrm{P} 300 \mathrm{BCl}$. A total of 23 subjects used a $\mathrm{P} 300 \mathrm{BCl}$ to spell the word "LUCAS" while receiving real-time, closed-loop feedback. The dry system yielded classification accuracies that were similar to those obtained with gel systems. All subjects completed a questionnaire after data recording, and all subjects stated that the dry system was not uncomfortable. This is the first field validation of a dry electrode P300 $\mathrm{BCl}$ system, and paves the way for new research and development with EEG recording systems that are much more practical and convenient in field settings than conventional systems.

Keywords: brain-computer interface, brain-machine interface, dry electrodes, gel electrodes, EEG, ERP, P300, intendiX.

\section{INTRODUCTION}

Brain-computer interfaces (BCIs) allow communication without movement. In a typical BCI, a user performs voluntary mental tasks that each produce distinct patterns of electrical activity in the electroencephalogram (EEG). Automated signal processing software tries to identify which mental tasks a user performed at specific times and thereby infer user intent. Most modern BCIs rely on one of three types of mental tasks, which are associated with different types of brain activity (Wolpaw et al., 2002):

Imagined movement, which produces event-related desynchronization (ERD; Guger et al., 2003; Pfurtscheller et al., 2006; Neuper et al., 2009; McFarland et al., 2010; Vidaurre et al., 2011); Attention to oscillating visual stimuli, which produces steadystate visual evoked potentials (SSVEP; Friman et al., 2007; Lin et al., 2007; Ortner et al., 2011; Allison et al., 2012);

Attention to transient stimuli, which produces the P300 eventrelated potential (ERP; Sellers et al., 2006; Zhang et al., 2008; Guger et al., 2009; Townsend et al., 2010; Jin et al., 2012).

Despite some delightfully strident arguments within the research community, there is no general agreement on which approach is best. Instead, different BCIs are better suited to different users, needs, environments, applications, and other parameters. The main drawback of the P300 BCI is that users must pay attention to specific events, usually flashes on a monitor. Hence, users must pace themselves according to the system, and may find the flashes annoying. On the other hand, P300 BCIs seem to work for nearly all healthy users, unlike (at least) ERD BCIs (Guger et al., 2003, 2009; Allison and Neuper, 2010). P300 BCIs require almost no training for the system or user, and have always been relatively fast; the first BCI to exceed $100 \mathrm{bits} / \mathrm{min}$ was a P300 BCI (Brunner et al., 2011). Recent work has highlighted many new options for improving the P300 BCI (e.g., Münßinger et al., 2010; Townsend et al., 2010; Frye et al., 2011; Kaufmann et al., 2011; Jin et al., 2012). Therefore, $\mathrm{P} 300$ BCIs should remain prominent for at least the near future.

Most P300 BCIs allow users to choose one target from several options, such as letters or numbers presented on a monitor (Farwell and Donchin, 1988; Townsend et al., 2010; Brunner et al., 2011). The user is asked to focus on one item, such as the letter "G," and count each time it flashes while ignoring other events. Next, different groups of letters briefly flash, in sequence, until the system has presented a prespecified number of targets (Farwell and Donchin, 1988) or accurate classification is possible based on the EEG data (Jin et al., 2012). Since counting each flash produces a brainwave called the P300, which is not elicited by ignored flashes, the system can identify the target letter by identifying which flashes elicited a P300. The system presents this letter to the user, and then moves to the next target.

However, like most BCIs, P300 BCIs are hampered by the need for conductive gel to get a good contact between each electrode and the scalp. Preparing a subject for conventional EEG recording requires abrading the skin under each electrode, positioning each electrode over the abraded area, and squirting electrode gel underneath each electrode. Getting a good contact between each 
electrode and the scalp usually requires further skin abrasion and application of gel. The gel is uncomfortable to many subjects, and must be washed out of both the cap and hair later. This procedure greatly increases the time and inconvenience needed for any EEG recording session. Also, after a few hours of use, the gel may dry, and new gel must be applied (Ko and Hynecek, 1974).

These problems reduce the appeal of EEG-based technologies to most users, and can be especially pronounced for severely disabled users - even though these are the people who need BCIs most. Some conditions can increase skin sensitivity, making the skin abrasion process more painful. Since some of these patients cannot communicate without a BCI, they may have no way to convey their distress during preparation. Hence, working with gel-based electrodes increases dependence on friends, family, or other caretakers.

Numerous recent articles that survey different end users have further confirmed that dry electrodes are a very high priority. Casson et al. (2010) surveyed neurologists and found that almost 90\% agreed there is a clinical need for "wearable electrodes." Huggins et al. (2011) surveyed 61 ALS patients and found one of their main concerns was "set-up simplicity." Zickler et al. (2011) surveyed severely disabled users and found that major issues included "possibility of independent use" and "easiness of use." Blain-Moraes et al. (2012) presented a focus group study with eight ALS patients and nine carers. Two of their main concerns were a more convenient way to sense brain signals, and facilitating independence for both users and carers. The future BNCI roadmap, an extensive analytical effort developed through many established BCI stakeholders through 2010 and 2011, identified "practical electrodes" as one of the two disruptive technologies in BCI research.

Since dry electrodes can substantially simplify EEG recording, and are identified as important across numerous surveys, there should be a strong interest in them. Indeed, numerous articles over many years have explored dry electrodes to record EEG and other physiological signals (e.g., Roman, 1966; Richardson, 1967, 1968; Ko and Hynecek, 1974; Taheri et al., 1994; Gevins et al., 1995). However, early dry electrodes had various problems including reduced signal quality, robustness to movement, electrical artifacts, cost, and comfort.

There are two general approaches to dry electrodes, each with distinct problems (Portnoy et al., 1974; Tam and Webster, 1977; Gargiulo et al., 2008; Volosyak et al., 2010). One approach uses capacitive sensors, which do not require direct contact with the scalp. For such systems, motion artifact has been an insurmountable problem so far. The second approach relies on penetrating the first layer of the skin, such as through micro-needles, bristle sensors, or mechanical springs that press electrodes into the skin (Taheri et al., 1994; Matteucci et al., 2007; Popescu et al., 2007; Grozea et al., 2011). These systems may be uncomfortable. And, with any dry electrode, a paramount concern is validating signal quality for a specific application in real-world circumstances.

Recently, interest in dry electrodes has increased considerably. A PubMed search on February 1, 2012 for "dry electrode EEG" revealed 37 articles; only five were published before 2000, and another five were published before 2007. An otherwise identical search that replaced "EEG" with "BCI" found six articles, all published 2007 or later. Many companies are also producing dry electrode systems. Most sales are simple toy systems that detect drowsiness or fatigue with one electrode, unlike more complex multi-electrode systems that detect conventional BCI signals such as P300, SSVEP, and ERD.

Of the six journal publications that include "EEG" and "BCI," there seems to be a dearth of P300 BCI work. Carabalona et al. (2009) focuses primarily on rehabilitation rather than dry electrodes. Popescu et al. (2007) only addressed motor imagery BCIs. Luo and Sullivan (2010) and Chi et al. (2011) only worked with SSVEP. Grozea et al. (2011) did explore P300s, but used an auditory paradigm, which is appealing to blind users but much less common and effective than a visual P300 approach. The article relied on only three electrodes: $\mathrm{Cz}$ and $\mathrm{P} 1$, referenced to Fz. This montage is not optimal for a visual P300 approach, which better works using components from parieto-occipital regions. Also, an Fz reference electrode is much more vulnerable to blink artifact than the more conventional mastoid or earlobe reference - and P300 BCIs are more vulnerable to eyeblink artifact than other BCIs. Although the article states that feedback was provided, it never mentions whether or how it was presented with the P300 BCI. Zander et al. (2011) explored ERPs and alpha activity. The ERP work used a completely novel display, unlike any P300 BCI yet. Their dry electrode system used only three electrodes, all located in a horizontal line. As with the Grozea et al. (2011) article, the additional spatial information available from a broader montage would have improved performance (e.g., Krusienski et al., 2006, 2008). Furthermore, Zander et al. (2011) was an offline study - subjects did not receive feedback, and classification accuracy was only estimated from offline data. Most importantly, their study showed that dry electrodes were statistically significantly worse (lower classification accuracy) than gel-based alternatives. Their classification accuracy was about $6 \%$ worse with the dry electrode system.

In addition to these journal publications, some conference papers have presented dry electrode systems. For example, our 2007 conference paper evaluated a dry electrode system in a gaming context, and a later conference paper used the same company's system in a P300 copy-spelling task (Trejo et al., 2007; Sellers et al., 2009). Both of these studies used relatively few subjects, did not report comfort, have never been translated to a journal paper, and reported poor performance. For example, the latter article reported only about $70 \%$ accuracy with a P300 BCI copy-spelling task using row-column flashes with a $6 \times 6$ matrix.

All of these factors underscore the opportunity and need to explore well-established P300 methods with dry electrodes. The primary goal of this study was to assess a new dry electrode system in an otherwise conventional P300 BCI. A total of 23 subjects used a P300 BCI that was very much like other P300 BCIs in terms of the display, task, paradigm, signal processing, and other details. We present conventional analyses such as accuracy as well as subjective report regarding the comfort of the dry electrode system.

\section{MATERIALS AND METHODS EXPERIMENTAL PROCEDURE}

Twenty-three subjects (six female, age: 22-60) participated in the study. All subjects were free of medication, had normal vision or vision corrected to normal, and no history of central nervous system abnormalities. All subjects provided informed consent before participating in the study. The procedure complied with 
the ethical review procedures within the BrainAble project. Subjects were prepared for recording with the dry electrode system, using eight recording sites plus a reference and ground as shown in Figure 1. Subjects were not seated in a shielded room, but an open office environment with periodic distractions, typical of a realworld setting. The entire preparation procedure took less than 5 min.

Subjects sat in front of a laptop computer and were instructed to relax and remain as still as possible. The laptop used the intendiX row/column (RC) speller shown in Figure 1 (g.tec medical engineering $\mathrm{GmbH}$, Austria). The RC speller presented 50 characters (the 26 letters of the English alphabet, integers from 0 to 9, and 14 special characters) on the laptop monitor. Subjects were instructed to silently count each time a target character flashed while ignoring other flashes. Subjects were first asked to spell the word "WATER" in an offline mode to help calibrate the system. Hence, the first target letter was "W." At the beginning of each trial, the target letter was highlighted for several seconds so the subject could identify the target. After a 2-s delay, the system then began flashing a randomly selected column or row for $100 \mathrm{~ms}$, followed by a $60-\mathrm{ms}$ delay before a different row or column is highlighted. Each row and column was highlighted 15 times for each letter. Therefore, there were 225 flashes per trial, and 1125 flashes for a five-letter word.

After each trial, the signal processing software extracted individual ERPs from $100 \mathrm{~ms}$ before to $700 \mathrm{~ms}$ after each flash. The 100-ms time segment before each flash was used for baseline correction. While subjects spelled the word "WATER," they did not receive any feedback. Next, the system performed linear discriminant analysis (LDA) based on these ERPs to create the weight vector for the upcoming online trials.

Next, the subject was asked to spell the word "LUCAS" in the same fashion as "WATER," except that the system used the updated classifier and provided real-time feedback. Only the results of the effort to spell "LUCAS" are reported in this paper. After each trial, the intendiX system presented the target character on the top of the monitor. The delay between the last row or column flash and the presentation of this feedback was less than $1 \mathrm{~s}$. After the feedback was presented, there was a delay of $2.15 \mathrm{~s}$ before the subject was cued to the next target character, and the highlighting sequence began again.
At the end of the recording procedure, some subjects chose to continue using the system in "free spelling" mode, in which they spelled text of their choosing. The data from these runs are not presented here, but Figure 1B presents one example of a subject spelling freely. When the subjects were done, the electrode system was removed. Unlike typical EEG recording sessions, it was not necessary to wash the subject's hair nor the electrodes to remove gel. Subjects completed a brief questionnaire before departing that asked about the discomfort produced by the electrodes.

After this procedure was complete, one of the 23 subjects then repeated the procedure using gel-based electrodes as described below. This additional recording was performed so we could present a direct, within-subject comparison of the raw EEG, ERPs, and BCI performance resulting from dry vs. gel electrodes, shown in Figures 3-5.

\section{HARDWARE AND SOFTWARE}

Figure 1 shows the electrode configuration and the electrode locations used for the studies. Figure 2 shows a close-up of the dry electrodes. There are two versions of the dry electrodes, which

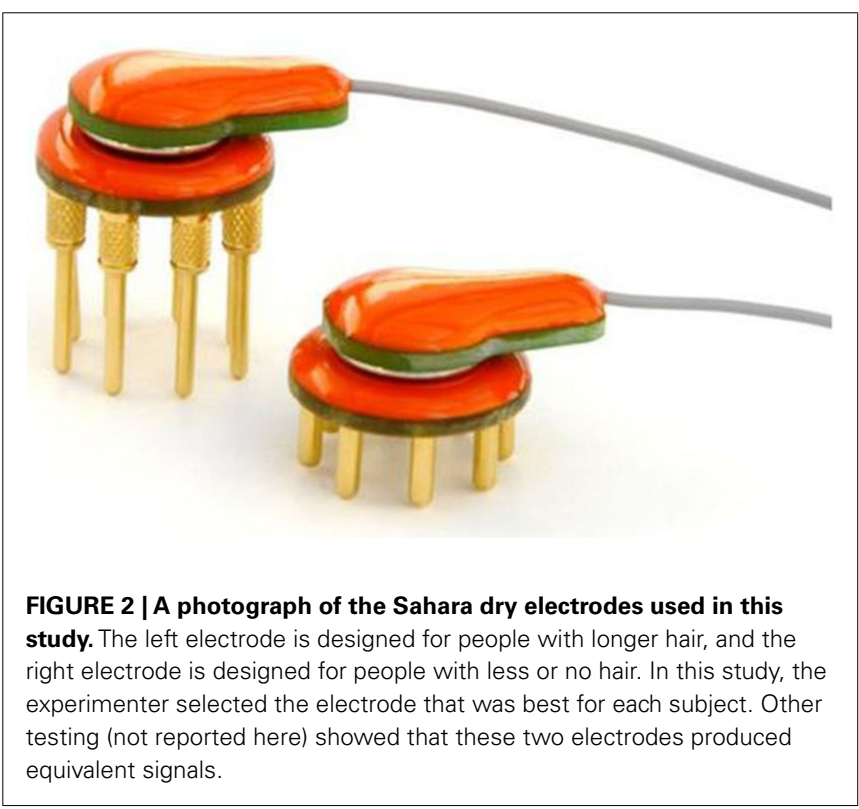

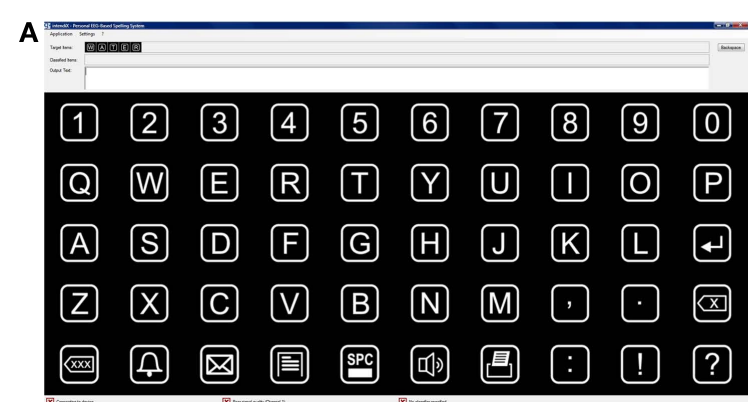
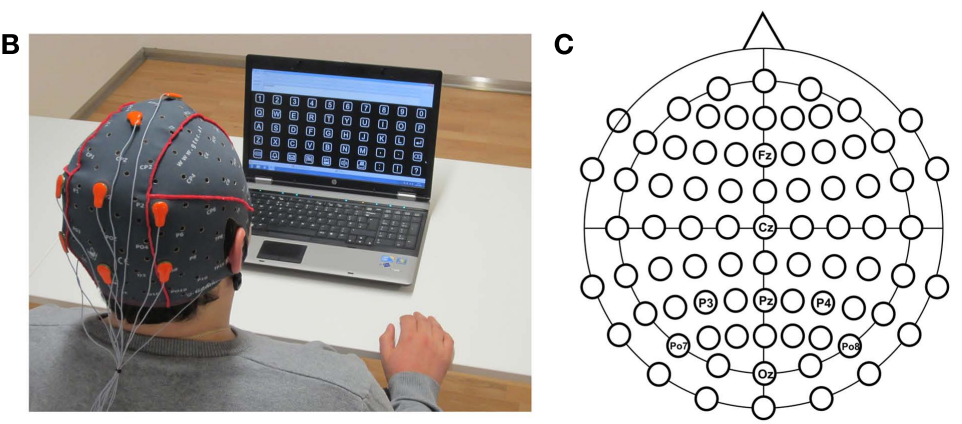

FIGURE 1 | (A) Shows the intendiX spelling matrix at the beginning of a "copy-spelling" run. The phrase "WATER" is presented at the top of the screen. The "W" is highlighted, indicating that it is the target letter. (B)
Presents a photograph of a subject using the system in free spelling mode. (C) Shows the electrode montage used with both the gel and dry electrodes. The ground is on the left mastoid, and the reference is on the right mastoid. 
differ in the length of the gold pins that contact the surface of the head. When preparing the subject for recording, the experimenter chose one of these electrodes based on the subject's hair length and head shape.

Electroencephalogram data were acquired using a g.USBamp (24 Bit biosignal amplification unit, g.tec medical engineering $\mathrm{GmbH}$, Austria) with a sampling frequency of $256 \mathrm{~Hz}$. The data were then converted to double precision, bandpass filtered between 0.5 and $30 \mathrm{~Hz}$, and then down-sampled to $64 \mathrm{~Hz}$. The ground electrode was mounted over the left mastoid and the reference was mounted over the right mastoid; for both positions disposable pre-gelled electrode pads were used. EEG electrodes were fixed to an EEG electrode cap (g.GAMMAcap) according to the extended international 10/20 electrode system. EEG recordings based on gel electrodes were done with the g.BUTTERfly electrode (golden ring electrode type with a hole in the middle to inject the gel); EEG recordings based on dry electrodes instead used the g.SAHARA electrode (eight gold-coated pins with $7 \mathrm{~mm} / 16 \mathrm{~mm}$ length mounted in a circular arrangement, diameter $15 \mathrm{~mm}$ ). Both types of electrodes are active EEG electrodes with a small preamplifier located in the electrode itself. Both types of electrodes do not penetrate the epidermis. The tips of the electrode contacts in the g.SAHARA system are smooth, not pointed, to avoid discomfort.

\section{RESULTS}

Before presenting results with the P300 speller, we first address the a priori question of whether the raw data from the two electrode types looked similar. Figure 3 presents $8 \mathrm{~s}$ of raw EEG data recorded from one subject with both gel and dry electrodes. The raw data look similar for both electrode types, including the noise created by eyeblink artifacts and some high frequency activity.
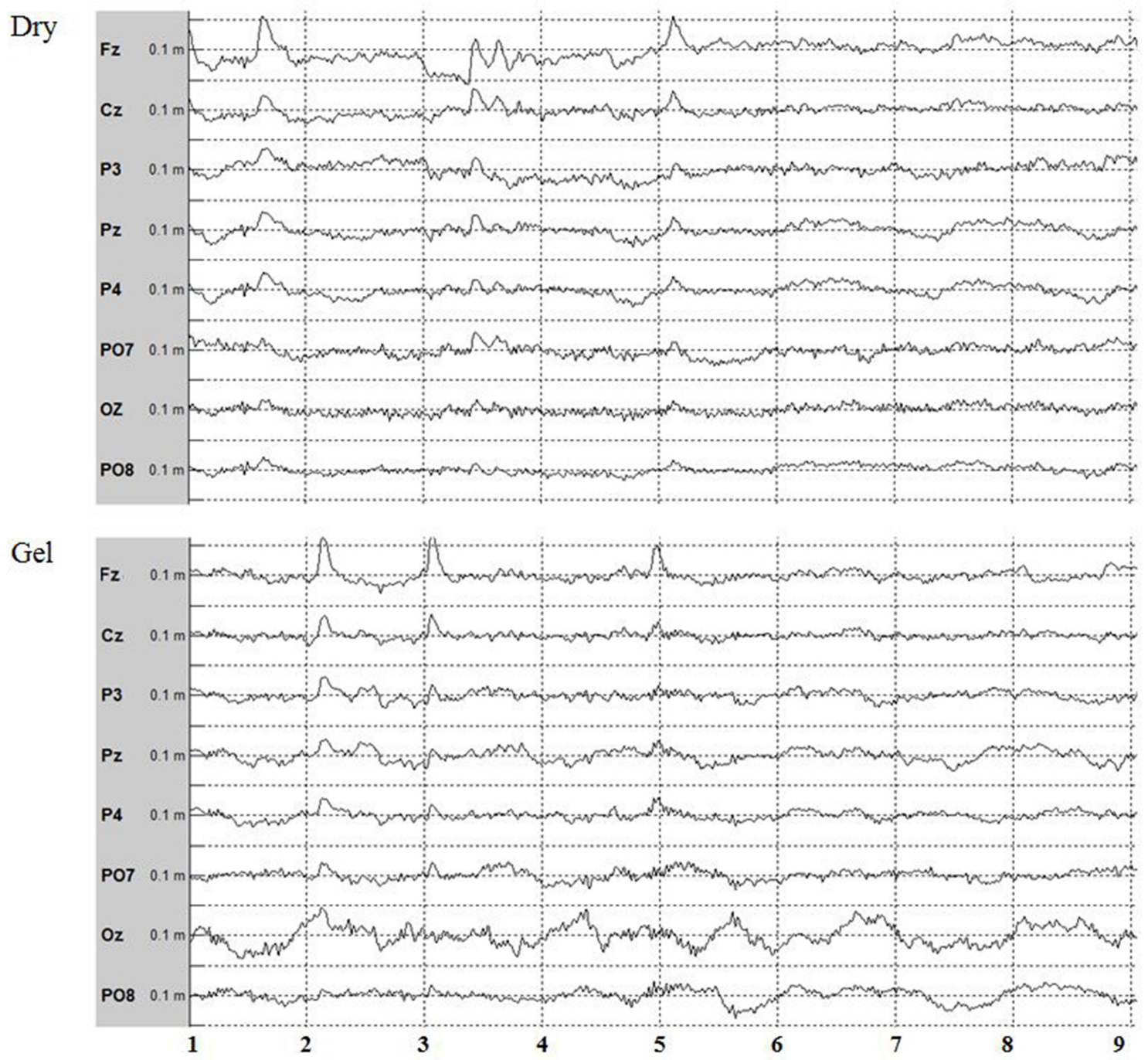

FIGURE 3 | Eight-channel EEG data from the P300 experiment acquired with dry and gel electrodes for one subject over frontal, central, parietal, and occipital sites. The EOG artifacts are mostly visible on $\mathrm{Fz}, \mathrm{Cz}, \mathrm{P} 3, \mathrm{Pz}$, and
P4 and look fairly similar for gel and dry electrodes. The $y$-axis is scaled with $\pm 100 \mu \mathrm{V}$, and the $x$-axis presents seconds. The data are bandpass filtered between 0.1 and $30 \mathrm{~Hz}$ with a $50 \mathrm{~Hz}$ notch filter. 
The next question is whether the ERPs look similar across both electrode types. Figure 3 shows the EP for dry and gel based electrodes at electrode position $\mathrm{Cz}$ for the training and copy-spelling run for one subject. The EP reaches its maximum of about $6 \mu \mathrm{V}$ after about $240 \mathrm{~ms}$ in both cases. The EP looks very similar for the dry and gel based electrodes and the comparison of the training and copy-spelling run shows that the EP is very stable over time.

\section{P300 BCI PERFORMANCE}

Table 1 summarizes the results of the current study. One column presents the results with dry electrodes, and another column summarizes results from a large group study with gel electrodes $(N=81$; Guger et al., 2009). We conducted a $t$-test to compare subjects' performance with gel vs. dry electrodes. We used the 81 subjects from the 2009 study who completed the row-column task and compared their accuracies to the 23 subjects from the present study. The difference was not statistically significant.

Table 1 clearly shows that the dry electrode system delivers performance comparable to the gel based system. However, it is important to establish whether other differences between the two studies might have created a bias toward the present study. Both studies used identical hardware from the same manufacturer, except for the electrodes. Both studies used very similar software, with no relevant differences in signal processing nor classifier updating. Both studies used the same sampling frequency, bandpass filter, and downsampling. In both studies, subjects spelled "WATER" to calibrate the system, then spelled "LUCAS" with feedback. In both studies, each row or column was highlighted for $100 \mathrm{~ms}$, followed by a delay of $60 \mathrm{~ms}$ before the next flash.

There is one noteworthy paradigmatic difference that affects classification accuracy. The 2009 study used a display with 36 characters. Hence, chance performance was one in 36 , or about $2.8 \%$. The present study instead had a vocabulary of 50 characters, corresponding to $2 \%$ chance performance. Therefore, in the 2009 study, correct classification due to chance was slightly more likely than in the present study.

The present study flashed each character 15 times, consistent with canonical work (Farwell and Donchin, 1988). However, we expect that the intendiX system could be practical with fewer

Table 1 | This table summarizes subjects' accuracy for gel electrodes in an earlier study (Guger et al., 2009) and dry electrodes in the present study.

\begin{tabular}{lll}
\hline $\begin{array}{l}\text { Row-column speller } \\
\text { classification } \\
\text { accuracy in \% }\end{array}$ & $\begin{array}{l}\text { Gel electrodes }(\boldsymbol{N}=\mathbf{8 1} ; \\
\text { Guger et al., 2009) }\end{array}$ & $\begin{array}{l}\text { Dry electrodes } \\
(\mathbf{N}=\mathbf{2 3})\end{array}$ \\
\hline 100 & & 69.6 \\
$80-100$ & 72.8 & 87.0 \\
$60-79$ & 88.9 & 8.7 \\
$40-59$ & 6.2 & 4.4 \\
$20-39$ & 3.7 & 0 \\
$0-19$ & 0.0 & 0 \\
Average accuracy of & 1.2 & $90.4 \pm 17.2$ \\
all subjects & $91.0 \pm 18.5$ & \\
\hline
\end{tabular}

$N$ specifies the number of subjects summarized in each column. flashes, and we want to facilitate comparison with other articles, which often present accuracy across different numbers of flashes to explore the speed-accuracy tradeoff (e.g., Farwell and Donchin, 1988; Townsend et al., 2010; Jin et al., 2012). Therefore, in addition to these results based on 15 flashes of each row and column, we also present accuracy with fewer flashes. Figure 5 presents accuracy across 1-15 different such flashes. Figure 5 shows that the dry electrodes compete well with the gel electrodes in this comparison as well.

\section{SUBJECTIVE REPORT}

All 23 subjects were asked whether the dry electrode system was uncomfortable in a short questionnaire. None of the subjects reported any discomfort through these questionnaires, nor did they complain in any other way. In the 2009 study none of the subjects reported any discomfort from the g.BUTTERFLY electrodes that were used.

Furthermore, g.tec hosts several workshops a year, in which hundreds of people have used g.BUTTERFLY electrodes, and dozens have used the g.SAHARA electrodes. None of these participants have reported any discomfort with either electrode. Hence, at least with the conditions used in this study and g.tec workshops, both electrode types were not uncomfortable.

\section{DISCUSSION}

This is the first journal publication to show that dry electrodes can yield performance comparable to gel electrodes with a P300 BCI. This is an important outcome, given the promise and prominence of both dry electrodes and P300 BCIs. Subjects did not consider the dry electrode system uncomfortable, and it required no gel and reduced preparation time. Although the dry electrode system might be more prone to movement artifact and ambient electrostatic charges, these were not a problem in the present study. Overall, the new system is generally comparable to or better than gel electrodes in many ways.

This work is also important because it demonstrates that the dry electrode system can function in a relatively unconstrained field setting. Dry electrodes typically entail much higher impedance values than gel electrodes. This is the main reason why gel is so common; the gel provides contact between the scalp and each electrode that greatly reduces impedance. Otherwise, high impedance will impair signal quality and increase vulnerability to electrical artifact, such as from external devices or movement. The dry electrode system allows good performance despite high impedances by using multiple gold-coated pins in each electrode, as well as an integrated amplifier within each electrode.

Interestingly, although the dry electrode system resulted in EEG data, ERPs, and BCI accuracy comparable to gel electrodes, there were notable differences. The average peak P300 amplitude was lower in the present study. The dry electrodes showed higher signal drifts below $3 \mathrm{~Hz}$ than gel based electrodes. Neither of these difference had a notable effect on classification accuracy. However, these differences might be relevant for other types of BCIs. For example, BCIs that rely on slow cortical potential changes (Birbaumer et al., 1999) might be more sensitive to signal drift below $3 \mathrm{~Hz}$.

Although this is a promising start, there are many issues that still need to be explored. As noted, dry electrodes need to be 


\section{Dry Electrode}

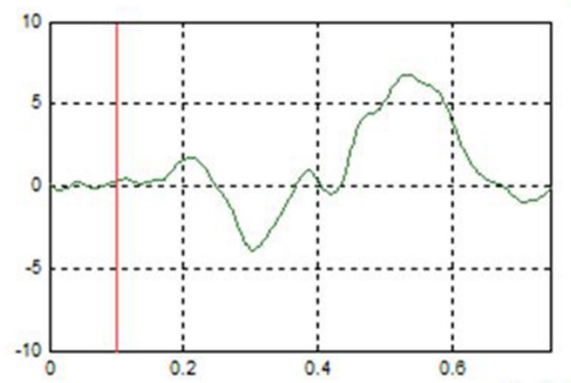

Copy Spelling
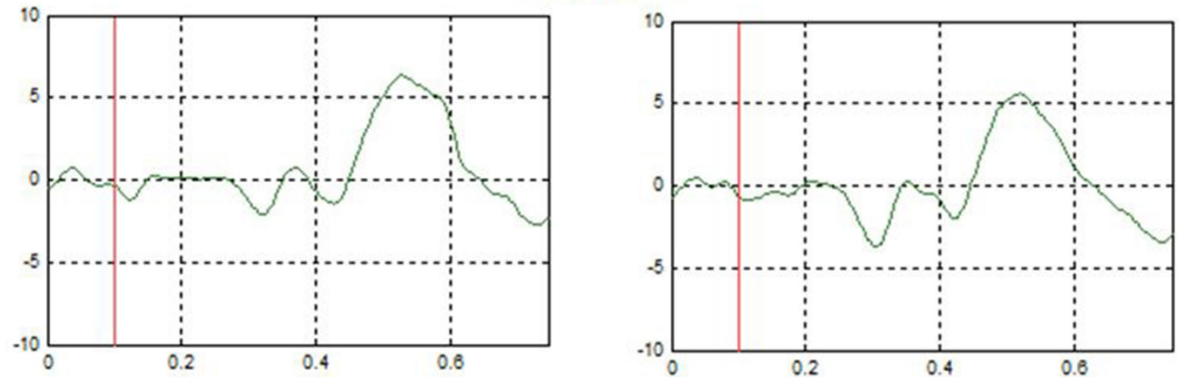

FIGURE 4 | The P300 complex recorded with dry and gel electrodes for one subject over site Cz. Each figure reflects averaged data across all target flashes for one five-letter word (150 flashes). The $y$-axis is scaled with $\pm 10 \mu \mathrm{V}$, and the $x$-axis in presented in seconds.

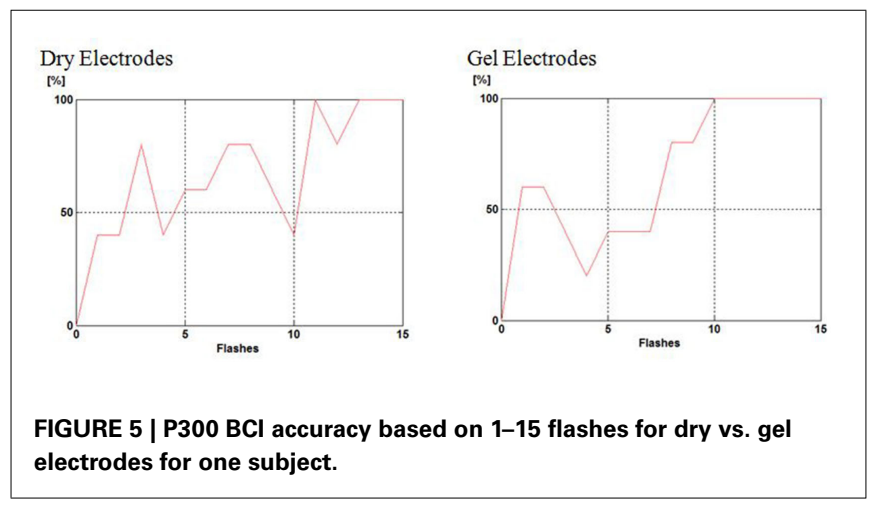

explored with a much wider variety of BCI systems, with different mental tasks, EEG signals, and other details. Comfort and other subjective factors should be assessed in other circumstances, such as with electrodes mounted in other headwear, tasks requiring

physical movement, and long-term use. All of the subjects in this study, like all published dry electrode studies, were healthy. Dry electrodes should be validated with persons with severe disabilities, since they often need BCIs more than healthy users. With some such users, special concerns (such as fasciculations that produce uncontrolled movement) might be especially problematic for dry electrodes. We also recommend focusing on other comparisons between dry and gel electrodes. While accuracy and comfort are very important, research should also parametrically compare preparation time, reliance on outside support, and other factors. These may be more difficult to paradigmatically assess, but are critical factors in BCI adoption (Allison, 2010; Casson et al., 2010; Huggins et al., 2011; Zickler et al., 2011; Blain-Moraes et al., 2012) and are worth the extra trouble.

\section{ACKNOWLEDGMENTS}

This work was funded by EC projects: CSI, ALIAS, Brainable, Decoder, and Better.

mediators of brain-computer interface user acceptance: focus group findings. Ergonomics. doi:10.1080/00140139.2012.661082.

Birbaumer, N., Ghanayim, N., Hinterberger, T., Iversen, I., Kotchoubey, B., Kübler, A., Perelmouter, J., Taub, E., and Flor, H. (1999). A spelling device for the paralysed. Nature 398, 297-298.

Brunner, P., Ritaccio, A. L., Emrich, J. F., Bischof, H., and Schalk, G.
(2011). Rapid communication with a "P300" Matrix Speller using electrocorticographic signals (ECoG). Front. Neurosci. 5:5. doi:10.3389/fnins.2011.00005

Carabalona, R., Castiglioni, P., and Gramatica, F. (2009). Brain-computer interfaces and neurorehabilitation. Stud. Health Technol. Inform. 145, 160-176.

Casson, A., Yates, D., Smith, S., Duncan, J., and Rodriguez-Villegas, 
E. (2010). Wearable electroencephalography. What is it, why is it needed, and what does it entail? IEEE Eng. Med. Biol. Mag. 29, 44-56.

Chi, Y., Wang, Y., Wang, Y., Maier, C., Jung, T., and Cauwenberghs, G. (2011). Dry and noncontact EEG sensors for mobile brain-computer interfaces. IEEE Trans. Neural Syst. Rehabil. Eng. 20, 228-235.

Farwell, L. A., and Donchin, E. (1988). Talking off the top of your head. Electroencephalogr. Clin. Neurophysiol. 70, 510-523.

Friman, O., Volosyak, I., and Gräser, A. (2007). Multiple channel detection of steady-state visual evoked potentials for brain-computer interfaces. IEEE Trans. Biomed. Eng. 54, 742-750.

Frye, G. E., Hauser, C. K., Townsend, G., and Sellers, E. W. (2011). Suppressing flashes of items surrounding targets during calibration of a P300-based brain-computer interface improves performance. $J$. Neural Eng. 8, 025024.

Gargiulo, G., Bifulco, P., Calvo, R. A., Cesarelli, M., Jin, C., and van Schaik, A. (2008). "A mobile EEG system with dry electrodes," in IEEE Biomedical Circuits and Systems Conference (2008), 273-276.

Gevins, A., Leong, H., Du, R., Smith, M. E., Le, J., DuRousseau, D., Zhang, J., and Libove, J. (1995). Towards measurement of brain function in operational environments. Biol. Psychol. 40, 169-186.

Grozea, C., Voinescu, C. D., and Fazli, S. (2011). Bristle-sensors - low-cost flexible passive dry EEG electrodes for neurofeedback and BCI applications. J. Neural Eng. 8, 025008.

Guger, C., Edlinger, G., Harkam, W., Niedermayer, I., and Pfurtscheller, G. (2003). How many people are able to operate an EEG-based braincomputer interface? IEEE Trans. Neural Syst. Rehabil. Eng. 11, 145-147.

Guger, C., Daban, S., Sellers, E., Holzner, C., Krausz, G., Carabalona, R., Gramatica, F., and Edlinger, G. (2009). How many people are able to control a P300-based brain-computer interface (BCI)? Neurosci. Lett. 462, 94-98.

Huggins, J. E., Wren, P. A., and Gruis, K. L. (2011). What would braincomputer interface users want? Opinions and priorities of potential users with amyotrophic lateral sclerosis. Amyotroph. Lateral Scler. 12, 318-324.

Jin, J., Allison, B. Z., Wang, X., and Neuper, C. (2012). A combined braincomputer interface based on P300 potentials and motion-onset visual evoked potentials. J. Neurosci. Methods 205, 265-276.

Kaufmann, T., Schulz, S. M. Grünzinger, C., and Kübler, A. (2011). Flashing characters with famous faces improves ERPbased brain-computer interface performance. J. Neural Eng. 8, 056016.

Ko, W. H., and Hynecek, J. (1974). "Dry electrodes and electrode amplifiers," in Biomedical Electrode Technology, eds H. A. Miller and D. C. Harrison (New York, NY: Academic Press), 169-181.

Krusienski, D., Sellers, E., Cabestaing, F., Bayoudh, S., McFarland, D., Vaughan, T., and Wolpaw, J. R. (2006). A comparison of classification techniques for the P300 Speller. J. Neural Eng. 6, 299-305.

Krusienski, D. J., Sellers, E. W., McFarland, D. J., Vaughan, T. M., and Wolpaw, J. R. (2008). Toward enhanced P300 speller performance. J. Neurosci. Methods 167, 15-21.

Lin, Z., Zhang, C., Wu, W., and Gao, X. (2007). Frequency recognition based on canonical correlation analysis for SSVEP-based BCIs. IEEE Trans. Biomed. Eng. 54, 1172-1176.

Luo, A., and Sullivan, T. J. (2010). A user-friendly SSVEP-based braincomputer interface using a timedomain classifier. J. Neural Eng. 7, 26010.

Matteucci, M., Carabalona, R., Casella, M., Di Fabrizio, E., Gramatica, F., Di Rienzo, M., Snidero, E., Gavioli, L., and Sancrotti, M. (2007). Micropatterned dry electrodes for braincomputer interface. Microelectron. Eng. 84, 1737-1740.

McFarland, D. J., Sarnacki, W. A., and Wolpaw, J. R. (2010). Electroencephalographic (EEG) control of three-dimensional movement. $J$. Neural Eng. 7, 036007.

Münßinger, J. I., Halder, S., Kleih, S. C., Furdea, A., Raco, V., Hösle, A., and Kübler, A. (2010). Brain painting: first evaluation of a new brain-computer interface application with ALS-patients and healthy volunteers. Front. Neurosci. 4:182. doi:10.3389/fnins.2010.00182

Neuper, C., Scherer, R., Wriessnegger, S., and Pfurtscheller, G. (2009). Motor imagery and action observation: modulation of sensorimotor brain rhythms during mental control of a brain-computer interface. Clin. Neurophysiol. 120, 239-247.

Ortner, R., Allison, B. Z., Korisek, G., Gaggl, H., and Pfurtscheller, G. (2011). An SSVEP BCI to control a hand orthosis for persons with tetraplegia. IEEE Trans. Neural Syst. Rehabil. Eng. 19, 1-5.
Pfurtscheller, G., Leeb, R., Keinrath, C. Friedman, D., Neuper, C., Guger, C. and Slater, M. (2006). Walking from thought. Brain Res. 1071, 145-152.

Popescu, F., Fazli, S., Badower, Y., Blankertz, B., and Müller, K. R. (2007). Single trial classification of motor imagination using 6 dry EEG electrodes. PLoS ONE 2, e637. doi:10.1371/journal.pone.0000637

Portnoy, W., David, R. M., and Akers, L. A. (1974). "Insulated ECG electrodes," in Biomedical Electrode Technology, eds H. A. Miller and D. C. Harrison (New York, NY: Academic Press).

Richardson, P. C. (1967). "The insulated electrode: a pasteless ECG technique," in Proceedings of the 20th ACEMB, Vol. 9, 157.

Richardson, P. C. (1968). "The construction techniques for insulated electrocardiographic electrodes," in Proceedings of 21st ACEM, Vol. 10, 13A1.

Roman, J. (1966). Dry electrodes for physiological monitoring. Tech. Note U S Natl. Aeronaut. Space Adm. D3414, 1-32.

Sellers, E. W., Krusienski, D. J., McFarland, D. J., Vaughan, T. M., and Wolpaw, J. R. (2006). A P300 eventrelated potential brain-computer interface (BCI): the effects of matrix size and inter stimulus interval on performance. Biol. Psychol. 73, 242-252.

Sellers, E. W., Turner, P., Sarnacki, W., McManus, T., Vaughan, T. M., and Matthews, R. (2009). "A novel dry electrode for braincomputer interface," in HumanComputer Interaction: Novel Interaction Methods and Techniques, ed. J. Jacko (Berlin: Springer Verlag), 623-631.

Taheri, B. A., Knight, R. T., and Smith, R. L. (1994). A dry electrode for EEG recording. Electroencephalogr. Clin. Neurophysiol. 90, 376-383.

Tam, H. W., and Webster, J. G. (1977) Minimizing electrode motion artifact by skin abrasion. IEEE Trans. Biomed. Eng. 24, 134-139.

Townsend, G., LaPallo, B. K., Boulay, C. B., Krusienski, D. J., Frye, G. E., Hauser, C. K., Schwartz, N. E., Vaughan, T. M., Wolpaw, J. R., and Sellers, E. W. (2010). A novel P300-based brain-computer interface stimulus presentation paradigm: moving beyond rows and columns. Clin. Neurophysiol. 121, 1109-1120.

Trejo, L. J., McDonald, N. J., Matthews, R., and Allison, B. Z. (2007). "Experimental design and testing of a multimodal cognitive overload classifier," in Automated Cognition
International Conference, Baltimore, MD.

Vidaurre, C., Sannelli, C., Müller, K. R., and Blankertz, B. (2011). Coadaptive calibration to improve BCI efficiency. J. Neural Eng. 8, 025009.

Volosyak, I., Valbuena, D., Malechka, T., Peuscher, J., and Gräser, A. (2010). Brain-computer interface using water-based electrodes. J. Neural Eng. 7, 066007.

Wolpaw, J. R., Birbaumer, N., McFarland, D. J., Pfurtscheller, G., and Vaughan, T. M. (2002). Braincomputer interfaces for communication and control. Clin. Neurophysiol. 113, 767-791.

Zander, T. O., Lehne, M., Ihme, K., Jatzev, S., Correia, J., Kothe, C. Picht, B., and Nijboer, F. (2011). A dry EEG-system for scientific research and brain-computer interfaces. Front. Neurosci. 5:53. doi: $10.3389 /$ fnins.2011.00053

Zhang, H., Guan, C., and Wang, C. (2008). Asynchronous P300-based brain-computer interfaces: a computational approach with statistical models. IEEE Trans. Biomed. Eng. 55, 1754-1763.

Zickler, C., Riccio, A., Leotta, F., HillianTress, S., Halder, S., Holz, E., StaigerSälzer, P., Hoogerwerf, E. J., Desideri, L., Mattia, D., and Kübler, A. (2011) A brain-computer interface as input channel for a standard assistive technology software. Clin. EEG Neurosci. 42, 236-244.

Conflict of Interest Statement: The Christoph Guger, Gunther Krausz, and Guenter Edlinger are full-time employees of g.tec medical engineering $\mathrm{GmbH}$, Guger Technologies OG, and the Christoph Guger and Guenter Edlinger are its co-CEOs.

Received: 08 March 2012; paper pending published: 26 March 2012; accepted: 09 April 2012; published online: 07 May 2012.

Citation: Guger C, Krausz G, Allison BZ and Edlinger $G$ (2012) Comparison of dry and gel based electrodes for P300 brain-computer interfaces. Front. Neurosci. 6:60. doi: 10.3389/fnins.2012.00060

This article was submitted to Frontiers in Neuroprosthetics, a specialty of Frontiers in Neuroscience.

Copyright (c) 2012 Guger, Krausz, Allison and Edlinger. This is an open-access article distributed under the terms of the Creative Commons Attribution Non Commercial License, which permits noncommercial use, distribution, and reproduction in other forums, provided the original authors and source are credited. 